DOI https://doi.org/10.24297/jap.v19i.9089/

\title{
Can Naked Singularities Exist Without Violating The Laws of Black Hole Thermodynamics?
}

\author{
Amal Pushp ${ }^{1,2}$ \\ ${ }^{1}$ Department of Physics, School of Basic Sciences, Manipal University Jaipur, Rajasthan 303007, India. \\ ${ }^{2}$ Torus Tech LLC, San Clemente, CA 92673, United States.
}

E-mail: amal@torustech.com

\begin{abstract}
According to the cosmic censorship conjecture, it is impossible for nature to have a physical singularity without a horizon because if it were to arise in any formalism, for instance as an extremal black hole (Kerr or Reissner-Nordstrom) then the surface gravity $\kappa=0$, which is a strict violation of the third law of black hole thermodynamics. In this paper we explore whether a true singularity can exist without defying this law.
\end{abstract}

Keywords: cosmic censorship conjecture, singularities, black hole thermodynamics.

\section{Introduction}

The study of the subject of gravitation since Newton, has led to enormous progress in our understandings of the intricate laws governing the universe. Einstein's realization that the nature of gravity is a geometrical one and that gravity by its very own nature is encoded in the curvature of space-time, has spurred major advances leading to a strong agreement between theory and observational data and continues to flourish even more than a century since general relativity was first theorized. In spite of the major successes of general relativity, there are still questions which are yet to be settled. One such question is whether space-time can conceive a true singularity unbounded with any horizon.

The concept of "singularity" arises in GR and is describable through the work of Penrose and Hawking in general relativity, the so called singularity theorems which holds true for any generic solution of Einstein field equations [1].

The cosmic censorship conjecture states that, the gravitational collapse of matter configurations will never generate a naked singularity [2]. In this note we are interested to reach a conclusion through the available data and inspect whether the conjecture can be falsified without leading to the violation of the third law of black hole thermodynamics. Let us begin with the preliminaries.

\section{Cosmic Censorship and Black Hole Laws}

The cosmic censorship follows from the following mathematical considerations. Consider a charged black hole solution with the Reissner-Nordstrom metric,

$$
d s^{2}=-\Delta d t^{2}+\Delta^{-1} d r^{2}+r^{2} d \Omega^{2}
$$

where,

$$
\Delta=1-\frac{2 G M}{r}+\frac{G\left(p^{2}+q^{2}\right)}{r^{2}}
$$

Here $M$ is the mass of the black hole, $q$ is the total electric charge and $p$ is the total magnetic charge. It is found that $r=0$ corresponds to a true singularity and the event horizon will occur at the radius where $\Delta$ vanishes which is given 
by,

$$
r_{ \pm}=G M \pm \sqrt{G^{2} M^{2}-G\left(p^{2}-q^{2}\right)}
$$

The above will have atmost three solutions. The first solution occurs at $G M^{2}<p^{2}+q^{2}$ in which the singularity occurs at $r=0$, a timelike line, hence there is no boundary condition which is materialized in the form of a physical horizon. The problem in having this is that the total energy of the black hole would be less than the contribution to the energy by the electromagnetic fields alone which means that the contribution from the mass would have to be negative inorder to cancel out the extra energy from the EM fields to fetch the exact energy of the hole. This anomaly marks the introduction of the conjecture as a sensible one. The second solution to equation (3) is given by $G M^{2}>p^{2}+q^{2}$ which is of interest to physicists as the solution is "physically realistic" ofcourse. The third solution would bring us to one of the central part connecting the main argument and is given by $G M^{2}=p^{2}+q^{2}$. The consequence of this solution is the extremal black hole. Jacob Bekenstein and Bardeen-Carter-Hawking (BCH) helped devise the four laws of black hole thermodynamics $[3,4]$. The law which is of interest to us in this paper is the third law, however, we will briefly state all the four laws and then stress on the third one in relation with the main argument.

*Zeroth law: The surface gravity, $\kappa$ of a stationary black hole is constant over the event horizon.

*First law: The first law can be summarised with the following formula,

$$
\delta M=\frac{\kappa}{8 \pi G} \delta A+\Omega_{H} \delta J
$$

*Second law: The area $\mathrm{A}$ of the event horizon of a black hole does not decrease with time, i.e., $\delta A \geq 0$.

* Third law: The surface gravity can never be zero or in other words it is impossible to achieve $\kappa=0$ in a finite sequence of operations.

The surface gravity of the black hole is given by the equation,

$$
\kappa=\frac{\sqrt{G^{2} M^{2}-a^{2}}}{2 G M\left(G M+\sqrt{G^{2} M^{2}-a^{2}}\right)}
$$

Consider for a moment that the magnitude of surface gravity for a black hole equals zero. This would then correspond to the case of extremal black holes where the emergence of singularities without boundary conditions is not improbable. However these black holes are only a theoretical possibility as of now and used as toy models for studying the intricacies of string theory and quantum gravity $[5,6]$.

It seems that extremal black holes will not satisfy our condition (formation of naked singularity without the violation of the third law of black hole thermodynamics). We have to search for a process of gravitational collapse which satisfactorily leads to a naked singularity and yet doesn't violate the third law. We'll thus have to look upon options other than extremal black holes.

Shapiro and Teukolsky have carried out numerical analysis arguing that sufficiently large spindle like matter configuration can lead to the formation of naked singularities in asymptotically flat spacetimes [7]. Quoting from their original paper,

"We find that the collapse of a prolate spheroid with sufficiently large semimajor axis leads to a spindle singularity without an apparent horizon. Our numerical computations suggest that the hoop conjecture is valid, but that cosmic censorship does not hold because a naked singularity may form in nonspherical relativistic collapse."

One thing is clear with this study that naked singularities "can" exist afterall. But what is not clear is that do these form under circumstances preserving the laws of black hole thermodynamics, specifically the third law? Moreover, is 
the preservation of the third law a necessary condition for a naked singularity to form? One can see that it is easier to answer the latter but the same is not true for the former given the availability of data. On a theoretical note, extremal black holes violate the third law inorder to falsify the cosmic censorship hypothesis. Therefore the third law is not a necessary criteria for naked singularities to exist in the case of extremal black holes but the same might not be true in the other cases involving true black holes. These ideas are worth pursuing further along these lines.

\section{Conclusions}

We can conclude by putting forward this observation: Naked singularity in the extremal limit cannot form without violating the third law of black hole thermodynamics so either extremal black holes do not really exist or we require a modification of the third law. In case the extremal black holes exist, the third law of black hole mechanics is not global in nature. For any other case of physically realistic black holes, the correctness of the third law of black hole thermodynamics is a necessary but not a sufficient condition for the formation of naked singularities.

In a recent paper it was shown that the violation of the cosmic censorship conjecture is impossible for extremal (as well as non-extremal) Kerr, Reissner-Nordstrom as well as Kerr-Newman Black Holes [8]. Although their results are based on a different set of parameters and constraints, the main idea is in close relation with what we have addressed in this note. The difference lies in the fact that we have arrived at the result by invoking black hole thermodynamics and our study is specific w.r.t. the extremal limit and does not say anything about the non-extremal case other than expressing a mere possibility of the violation of CCC for the same. A more explicit demonstration of our results is beyond the scope of this paper and would be addressed in a follow-up, possibly by advancing on this new theme provided by [8].

\section{References}

[1] R. Penrose, "Gravitational collapse and space-time singularities", Phys. Rev. Lett. 14 (3): 57 (1965). https://doi.org/10.1103/PhysRevLett.14.57

[2] R. Penrose, "Gravitational collapse: The role of general relativity", Nuovo Cimento Rivista Serie 1: 252-276 (1969).

[3] J. D. Bekenstein, "Black holes and the second law", Lettere al Nuovo Cimento 4 (15): 99-104 (1972). https://doi.org/10.1142/9789811203961_0022

[4] J. M. Bardeen, B. Carter \& S. W. Hawking, "The four laws of black hole mechanics", Commun. Math. Phys. 31: 161-170 (1973). https://doi.org/10.1007/BF01645742

[5] Steven B. Giddings, "Toy Models For Black Hole Evaporation", http://arxiv.org/abs/hep-th/9209113 (1992).

[6] A. Sen, "Extremal Black Holes And Elementary String States", Mod. Phys. Lett. A(10):2081-2093 (1995). https://doi.org/10.1142/S0217732395002234

[7] Stuart L. Shapiro, Saul A. Teukolsky, "Formation of naked singularities: The violation of cosmic censorship", Phys. Rev. Lett. 66: 994 (1991). https://doi.org/10.1103/PhysRevLett.66.994

[8] H. Khodabakhshi, F. Shojai, "Cosmic censorship conjecture in a general Kerr-Newman black hole", Annals of Physics 420 (2020). http://arxiv.org/abs/2008. 02358

\section{Conflicts of Interest}


The author declares that there are no conflict of interests.

\section{Funding Statement}

Acknowledgments

I would like to thank Olivier Alirol for fruitful discussions. 